\title{
Asymptomatic COVID-19; We Don't Know What We Don't Know
}

Olen R. Brown, Professor Emeritus, John M. Dalton Cardiovascular Research Center, University of Missouri-Columbia, MO.U.S.A.; BrownO@missouri.edu

\begin{abstract}
Decisions affecting the COVID-19 pandemic, by the individual and those with highest authority, are being made on the basis of unreliable data. Data about cases and deaths are collected daily but represent only a sample of reality. Statistics convert sample data into more reliable estimates. However, statistics have no magical powers; reliability requires dependable data. It is futile to rail against this darkness; COVID-19 is not a scientific experiment. However, we must do better both with data collection and data analysis. In this review, I focus on one element of the data, the asymptomatic case of COVID-19. Without reliable information about this number, decision makers are significantly blinded. By its nature, the asymptomatic case is hidden but contaminating to understanding COVID-19. The true case rate and death rate per case are unknowable without knowing the fraction of cases that are asymptomatic. The best estimate of asymptomatic cases is in the CDC document: COVID-19 Pandemic Planning Scenarios. For four different scenarios the estimates range from $10 \%$ to $70 \%$, with the best estimate of $40 \%$ for asymptomatic cases. However, even the definition of the asymptomatic case is problematic. In simplest terms, two elements are required: an infection and no symptoms. How is "no symptoms" to be usefully defined? It appears to be analogous to pontificating about black swans from studying only white swans. It implies infection, but how is infection defined? Is it presence of the virus, replication of the virus, or presence of antibodies? Is asymptomatic disease an oxymoron? Without extensive, purposeful screening for specifically defined, essential symptoms and appropriate virus and antibody testing over time, the class of asymptomatic cases remains unknown. Current estimates range from $<20 \%$ to $>80 \%$. If low, it can be ignored; if high, it dramatically and proportionately lowers the case rate and the death rate per case. Consequentially, the asymptomatic rate dramatically affects our societal and political responses. In this focused review, we assess the limitations of the published estimates, bring attention to the importance of obtaining accurate data, and exhort that high priority be given in the scientific community to understanding the issue, asymptomatic COVID-19 cases.
\end{abstract}

Keywords: asymptomatic disease; communicability; COVID-19; death rate; Ro; SARS-CoV-2; social distancing; transmission rate; infection rate; quarantine

\section{INTRODUCTION}

The rapid, world-wide spread of COVID-19 required that decisions to limit its communicability be made on the basis of scant information. It was rapidly determined that the outbreak was caused by a new (not previously identified) virus, SARS-CoV-2. The International Committee on 
Taxonomy of Viruses concluded that the virus was "completely independent" from the outbreak of SARS-CoV in 2002-2003 and they further stated ${ }^{1}$ :

It is thus reasonable to assume that this biased knowledge of the natural diversity of the species Severe acute respiratory syndrome-related coronavirus limits our current understanding of fundamental aspects of the biology of this species and, as a consequence, our abilities to control zoonotic spillovers to humans.

This limited understanding continues and seriously complicates efforts to define the disease and possibly to control the spread of the virus. The specific data-limitation addressed by the current focused review is the lack of information about asymptomatic cases.

In an article ${ }^{2}$ accepted June 4, 2020 and published by Cambridge University Press in the Journal Epidemiology and Infection, Zhao et al. states: “...the ratio of asymptomatic infection is a useful indicator of the burden of disease and a better measurement of the transmissibility of the virus. So far, people have not paid enough attention to asymptomatic carriers."

COVID-19 information is assimilated and published daily. Commonly, the data are reported as the number of cases based on population and the number of deaths per case. Clearly, to the extent that asymptomatic cases exist these data would be skewed- they would be wrong by overestimating deaths per case and underestimating the number of cases. If asymptomatic cases are relatively few the problem is minimal; if asymptomatic cases are frequent, the problem could make the computed case occurrence and case death rates no better than guesses. Because policy decisions designed to control the pandemic have such detrimental effects on economics and social behaviors, the most careful assessment possible of the extent to which values for the number of cases is contaminated by asymptomatic cases is obviously essential.

The difficulties in "managing" COVID-19 is exemplified in a report by Faranda et al. ${ }^{3}$. They stated:

COVID-19 is currently affecting over 180 countries worldwide and poses serious threats to public health as well as economic and social stability of many countries. Modeling and extrapolating in near real-time the evolution of COVID-19 epidemics is a scientific challenge, which requires a deep understanding of the non-linearity's undermining the dynamics of the epidemics. However we show that real-time predictions of CoVID-19 infections are extremely sensitive to errors in data collection and crucially depend on the latest available data points... Our goal is to show how uncertainties arising from both poor data quality and inadequate estimation of model parameters (incubation, infection, and recovery rates) propagate to long-term extrapolations of infection counts. We provide guidelines for reporting those uncertainties to the scientific community and 
the general public... Despite the importance of having robust estimates of the asymptotic total number of infections, early estimates of COVID-19 show enormous fluctuations... predictions are extremely sensitive to the reporting protocol and crucially depend on the last available data point before the maximum number of daily infections is reached... Our results suggest that there are physical and statistical reasons to assign low confidence to statistical and dynamical fits, despite their apparently good statistical scores.

The word asymptomatic itself has been (sometimes justifiably) used in confusing ways. An infectious condition in humans with no symptoms could exist, but it likely would not be recognized and how could it be called a disease? For infection of a population by a virus or bacterium, when does the seriousness and prevalence of symptoms qualify it as a disease? Is qualification dependent on prevalence or on seriousness? Has this ever been addressed quantitatively? However, scientists have attempted to apply the most powerful and sophisticated statistics to calculate the prevalence of asymptomatic COVID-19. Necessarily, an asymptomatic case of infectious disease is identified because the infectious agent is found to be present. Before there existed any means of culturing germs as agents of disease, the presence of characteristic signs and symptoms was used to define cases. How valid can be the early reports of case rates and death rates for COVID-19?

Identifying asymptomatic COVID-19 requires quantitative information for two elements: a case without symptoms and proof of infection, both within a relevant time frame. For COVAD-19, the prevalence of asymptomatic cases is fundamentally possible because sophisticated tests are available to detect the virus, or immune status as a response, in people with and without identifiable symptoms. The identifiable symptoms list for COVID-19 has evolved. Thus, what we mean by asymptomatic COVID-19 has changed over the short span of approximately six months since the first case was identified. As the symptoms recognized for COVID-19 increase, the number of recognized asymptomatic cases declines proportionately. The extent and nature of symptoms required for a non-asymptomatic case has not been consistent.

A curious result stems from this imprecision in definition; it results in imprecise thinking. The real quantity of asymptomatic and symptomatic cases is a specific number. In the real world the relationship between symptomatic and asymptomatic cases is fixed (barring changes in the virus or human population). As the number in either category increases, the number in the other category decreases by the same amount. This seems obvious; however, as the asymptomatic cases go to zero, the very definition of asymptomatic cases vanishes, and if all cases produce symptoms, the term has no practical use. With no asymptomatic cases, a case, or no case, can be defined only by signs and symptoms and the measurement of the presence or 
absence of the infectious agent is of little or no interest for assessing prevalence of the disease. I say this to propose that the categorization "asymptomatic" serves us poorly.

As scientists and physicians, I propose we should seek consensus to move to quantification of COVID-19 by seriousness of signs and symptoms with a range of categories carefully and specifically defined. Thus, each case of COVID-19 would be assigned to a category defined by serious consideration of the consequence for the patient. We would then not have the confusing statistics of: asymptomatic cases, symptomatic cases, total cases, and deaths. It should be decided carefully, but a reasonable measure of the effect on the infected individual and the associated effect on society (behavior, economics) could be devised. This surely would be based on a defined measure of the symptoms.

This idea can be examined by considering a generic example without using COVID-19. Suppose an infection by agent $X$ is detectable by a scientific test and that the infection produces no detectable harmful effects. Neither the prevalence nor spread of this condition (we should not call it a disease) would be of practical interest. Individuals and societies would not respond. Now, imagine that infection by $X$ does cause detectable symptoms and the symptoms are sufficient to identify the infection. Symptoms is a vague term, both regarding its usefulness in specifying a specific disease and for assessing its "seriousness" either to an individual or to a society. The rational response of individuals and societies would be proportional to its prevalence and "seriousness". This seems to be so fundamental as to not require stating. However, we have found it necessary because of the circumstances of COVID-19 to proceed with measures including lock-down, quarantine and social distancing without assessing the threshold of knowledge of seriousness and prevalence for COVID-19. The point is: we are now at the time-point where we must analyze the relevant data and make an informed decision about the reasonable societal response. This requires that the asymptomatic case be recognized and defined with the importance it deserves.

Death rate is currently estimated; the term is definitive; the assignment to COVID-19 is problematic. Surely society would not undergo the maximum control possible for a disease with symptoms of the common cold. COVID-19 may be the first disease where the societal and economic impacts have been either possible or implemented to the degree now seen. It will not be the last for which such considerations must be made. If a democracy can decide to make rules that regulate (to pick an arbitrary, non-controversial example) the concentration of carbon dioxide in the atmosphere, we can do better than decide emotionally, or based on deaths as a percentage of the total population, or on symptoms with no clear understanding of the extent of symptoms.

Today, with the current state of inadequate knowledge, the error in societal response to the pandemic should be on the conservative (prudent) side of choices. With the present course, 
however, over time we will move to persistence of, changes in, or abandonment of current policies. Current assessments of COVID-19, to the extent that they are not based on logic and are contaminated by serious misunderstandings of flawed data, must be improved to the maximum extent possible.

Because data about asymptomatic COVID-19 cases are limited, we have also included assessment of information for flu and SARS-CoV. This is done to focus attention on the serious problem with current incident and death rates for COVID-19; to urge caution in its application for policy decisions; and to encourage efforts to rapidly increase gathering of reliable data about asymptomatic cases. This can be done by appropriately-designed future tests for antibodies in populations and of appropriate use of data that is currently accumulating. The value is obvious. Some asymptomatic cases certainly occur. To the extent that they occur, and are not appropriately accounted for, we are "flying blind" in application of economic and socially significant regulations.

\section{OVERVIEW}

\section{The literature Reviewed}

The literature specific to COVID-19 asymptomatic cases is currently modest, but rapidly changing on a daily basis. A search using Ovid MEDLINE (until July 02, 2020) yielded 9541 articles for "COVID-19" and 1750 articles for "asymptomatic infections", and 70 articles for the terms combined by AND. I initially selected 26 relevant articles as the base and both expanded and limited this to focus on the significance of asymptomatic cases. I have selectively included limited data for flu and SARS-Co-V for context.

\section{Some Definitions}

Historically, it was usual to define a case based on diagnosis by signs and symptoms and this was improved when specific tests became available. For COVID-19, identification of SARS-CoV-2 virus was the confirming test from the beginning. The definition of symptoms has evolved and additional symptoms are now recognized; therefore, there is no specific definition which can be applied to the term symptomatic. Therefore, asymptomatic suffers also from lack of specific definition. Death appears to be a definitive criterion; however, cause of death is not and it is my opinion that deaths caused by other than SARS-CoV-2 have been included. It is recognized that imperfections exist in all data and with a sufficient data base, small discrepancies are tolerated.

\section{Perspectives about Asymptomatic Cases}

An article by Wang et al. ${ }^{4}$ stated: "On 31 March 2020, Chinese Health Authorization announced that numbers (sic) of asymptomatic cases with severe acute respiratory syndrome coronavirus 
2 (SARS-CoV-2) infection will be made to the public daily." The authors gave the characteristics of asymptomatic cases and described proposed guidelines for management of cases. The article apparently suffers from translation from Chinese into English. I shall paraphrase to provide maximum accuracy but with my interpretations. They stated that an asymptomatic infection is also known as a hidden or covert infection. Asymptomatic carrier was said to refer to a patient who has "mild or non-symptom" but has a positive test for the viral nucleic acid of SARS-CoV-2 or a positive test for serum specific immunoglobulin $\mathrm{M}$ antibodies. They indicate that the protocol of the National Health Commission of the People's Republic of China provides this information. The authors then explain that there are two classes of asymptomatic cases with SARS-CoV-2 infection:

[There are] cases with little or mild symptoms within the incubation period, but with symptoms onset in the coming 14-day quarantine period or typical changes on the chest-radiograph. [Some patients] had no symptoms all the time but tested positive for viral nucleic acid or antibodies. .. Currently, asymptomatic cases are not included in the confirmed patients in everyday-report according to the 'Novel Coronavirus Pneumonia Diagnosis and Treatment Protocol'... Once found, the patient will be put into medical isolation for up to 14-days, if the patient has symptoms onset within the isolation period, he/she will be reported as a confirmed case immediately.

The authors attempt to address the question of the number of asymptomatic cases. They cite evidence from the Diamond Princess ship and other evidence. They concluded: "With the existence of disagreement in the actual number of asymptomatic cases and their infectivity, larger observational studies including serological tests with a longer period are needed."

Oran and Topol ${ }^{5}$ recently brought attention to the prevalence of asymptomatic SARS-CoV- 2 infections: "It has been suspected that infected persons who remain asymptomatic play a significant role in the ongoing pandemic, but their relative number and effect have been uncertain." The authors conclude: "The likelihood that approximately $40 \%$ to $50 \%$ of those infected with SARS-CoV- 2 will remain asymptomatic suggests that the virus might have greater potential than previously estimated to spread silently and deeply through human population." They cite the "dual nature" of the disease: "...tragically lethal in some persons and surprisingly benign in others". A presumptive asymptomatic carrier state transmission in Wuhan, China was claimed by Bai et al. to have been the first to be reported ${ }^{6}$ and the person transmitted it to five relatives and the authors commented: "If the findings... are replicated, the prevention of COVID-19 infection would prove to be challenging."

In an article ${ }^{7}$, received for publication as a brief Letter February 13, 2020, the asymptomatic ratio of novel coronavirus infections was estimated (using case data for the Japanese national who was evacuated from Wuhan, China) with the conclusion: "Despite the small sample size, the estimation indicates that perhaps less than half of COVID-19-infected individuals are asymptomatic." The authors also concluded: "There is great need for further studies on the prevalence of asymptomatic COVID-19 
infections to guide epidemic control efforts." This ratio is somewhat smaller than that for influenza, which has been estimated at $56-80 \%^{8}$.

There has been speculation about the clinical significance of various viral infections ${ }^{9}$ : "Asymptomatic, chronic viral infections occur in a large portion of humanity... It is important to recognize that the study of asymptomatic chronic viral infections is an emerging field, and part of our discussion will necessarily be speculative." They reference an article that indicates that on the average, humans are concurrently infected with 8 to 12 viruses and the mechanisms that allow and perpetuate viral infections are poorly understood. These authors state a concept that is most relevant to the current review of asymptomatic COVID-19: "A detailed understanding of asymptomatic chronic viral infections is critical to analyze their pathogenesis, assess the severity and burden of disease and, where required, optimize public health control measures" ${ }^{9}$. This raises the specter that COVID-19 might produce chronic, asymptomatic carriers.

\section{Problems in Laboratory Diagnosis}

The gold standard for diagnosing CoviD-19 is real-time reverse transcriptase-polymerase chain reaction (rRT-PCR) based assays performed on respiratory specimens; however, point-of-care technologies and serologic immunoassays are rapidly emerging with high sensitivity and specificity. It is recognized that “... critical gaps still remain in screening asymptomatic people who are in the incubation phase... as well as accurate determination of live (sic) viral shedding during convalescence ..." ${ }^{10}$. It is apparent that the word asymptomatic has not always been used with the same meaning, and this has produced some of the variability seen in reported asymptomatic rates. However, the issue of the transmissibility of COVID19 from symptomatic vs. asymptomatic cases is surely relevant.

\section{Asymptomatic Carriers}

Zhao et al. ${ }^{2}$ in June, 2020 brought attention to the problem presented by the asymptomatic carrier state of COVIE-19 and concluded "So far, people have not paid enough attention to asymptomatic carriers". Particular attention was focused on the timeline of onset of symptoms in a familial cluster of six cases in China. The index case was positive by RT-PCR test without symptoms and normal chest computed tomography (CT) for 19 days which was said to be "comparatively long, but within the reported maximum incubation of 24 days". Data is presented about the sequence of spread of infections and the authors concluded: "... the asymptomatic carrier had a relevant epidemiologic history and it may be concluded that there is an asymptomatic spreader of COVID-19." They cite a report by Chen Yi et al. ${ }^{11}$ stated to be "... the first published quantitative study on the infectiousness of the asymptomatic transmission". They reported on 59 imported infectious patients (51 asymptomatic cases and eight asymptomatic carriers). A total of 2147 close contacts of these individuals were investigated, and 132 local residents were infected (110 symptomatic cases and 22 asymptomatic cases). The total infection rate was $6.15 \%$. Asymptomatic infections accounted for $16.7 \%$ of 2147 infections.

Wu ${ }^{12}$ inferred that 126 cases were transmitted from asymptomatic cases and six cases were transmitted from asymptomatic cases. Wu further concluded that on average, each symptomatic case patient could 
transfer the virus to approximately three other individuals while each asymptomatic carrier infected less than one other individual.

A family cluster of asymptomatic cases was investigated ${ }^{13}$. In a family of three, one had clinical symptoms including a decreased lymphocyte count, abnormal chest CT images, and a positive result on qRT-PCR. Two family members were both asymptomatic, with normal lymphocyte counts and chest CT images but positive qRT-PCR tests.

Pan et al. ${ }^{14}$ identified 26 persistently asymptomatic patients with positive test results for SARS-CoV-2 nucleic acid to determine the clinical characteristics and asymptomatic carrier transmission of COVID-19 infections. Twenty-two patients (84.6\%) selected as "correlated with clustering occurrence" were studied. The median period from contact to diagnosis and the last positive nucleic acid test was 19 (8-24 days) and 21.5 days (10-36 days), respectively. The median period from diagnosis to negative nucleic acid test was significantly different between patients with normal or atypical chest computed tomography (CT) findings ( $n=16,61.5 \% ; 7.5$ days [2-20 days]) and patients with typical ground-glass or patchy opacities on $C T$ ( $n=10,38.5 \% ; 12.5$ days [8-22 days]; $P<0.01$. Seven patients (70\%) with initial positive nucleic acid test results had a negative result simultaneously with improved CT findings.

Obvious improvement in CT findings was observed in three patients $(30.0 \%)$ despite positive nucleic acid test results. The authors concluded: "In asymptomatic patients, changes in biochemical and inflammatory variables are small and changes on chest CT can occur... the long existence of SARS-CoV-2 in some asymptomatic patients and false-negative results need to be considered in SARS-CoV-2 nucleic acid test."

This underscores the need for caution regarding published accounts of asymptomatic percentages, the urgent need for careful reanalysis of current estimates, and for future studies done appropriately with statistical analyses.

The question of the transmissibility of COVID-19 from asymptomatic carriers has received considerable attention because of its importance in attempted reduction of transmission. An early study used data from the city of Ningbo, China with chi-square tests predominately used to produce "contingency tables with low cell counts that included zero". It can be argued that this invalidates the statistics. The Ningbo data were reanalyzed ${ }^{15}$ using what the authors called "more appropriate methods" to obtain "more reliable and sound conclusions on the transmission rates of the coronavirus by the symptomatic and asymptomatic patients." The authors reported: "After excluding the cases related to the super-spreader, the Fisher exact test yields a $P$ value of .84, which indicates stronger evidence of no difference in the transmission rates compared with the original analysis." The odds ratio of the coronavirus transmission rates between symptomatic and asymptomatic patients was 1.2 with a $95 \%$ confidence interval 0.5-2.8. There was no difference in the transmission rates of coronavirus between symptomatic and asymptomatic patients.

The question of the clinical outcome for asymptomatic patients has been addressed ${ }^{16}$. The epidemiological and clinical outcomes were determined for 55 asymptomatic carriers who were laboratory confirmed to be positive for SARS-CO-V-2 by nucleic acid testing of pharyngeal swab samples. 
"The asymptomatic carrier seldom occurred among young people (aged 18-29 years) who had close contact with infected family members. In the majority of patients, the outcome was mild or ordinary 2019 novel coronavirus disease during hospitalization." Characteristics of 55 asymptomatic patients was tabulated by "infection classification" of mild (25.5\%), ordinary $(70.9 \%)$, and severe $(3.6 \%$ - all were $>60$ years old), and listed by age, sex, whether native to Hubei Province, and underlying diseases (hypertension, hypothyroidism, chronic pharyngitis, hepatitis B, asthma, and Cholecystitis).

The epidemiology and clinical characteristics of 7 cases of COVID-19 plus one case of close contact (8 total cases) in a "family aggregation epidemic" in Gansu Province were analyzed and reported ${ }^{17}$. The time-course of development of symptoms and diagnosis, whether symptomatic or asymptomatic, was recorded. Five cases were described as asymptomatic.

The proportion of pre-symptomatic transmission (and other factors) was calculated using outbreak data from clusters in Singapore and Tianjin, China ${ }^{18}$. Various factors were determined including the proportion of pre-symptomatic transmission which was $48 \%$ (95\% Credible interval: $32-67$ ) for Singapore and $62 \%$ (95\% credible interval: 50-76) for Tianjin. They concluded that "High estimates of the proportion of pre-symptomatic transmission imply that case finding and contact tracing need to be supplemented by physical distancing measures in order to control the COVID-19 outbreak." They noted that quarantine and other containment measures in place during data collection "may inflate the proportion of infections from pre-symptomatic individuals."

\section{Comparison of Asymptomatic and Symptomatic Cases}

A report dated June 23, 2020 was titled: The coronavirus crisis: we still don't fully understand the label 'Asymptomatic'. The introductory statement was: "Even if someone is infected by the novel coronavirus and remains asymptomatic - free of coughing, fever, fatigue and other common signs of infection... The virus can still cause mild - although likely reversible - harm to their lungs."

This report provides an account available to the general public of science that was unfolding and in this instance was published in Nature Medicine on June 18, $2020^{19}$. The science article was self-described as "Clinical and immunological assessment of asymptomatic SARS-CoV-2 infections". A confirmed case of COVID-19 was defined as: "an individual; with nasopharyngeal at swabs that were positive for SARS-CoV2, using laboratory-based PCR". The symptomatic patients were defined as "patients with laboratoryconfirmed COVID-19 with symptoms such as fever, cough, sore throat and sputum". An asymptomatic case was defined as an individual with a positive nucleic acid test result but without any relevant clinical symptoms in the preceding $14 \mathrm{~d}$ and during hospitalization". The methods were provided in detail. The work was introduced with the statement "The clinical features and immune responses of asymptomatic individuals infected with severe acute respiratory syndrome coronavirus 2 (SARS-Co-V) have not been well described". Asymptomatics (37 asymptomatic individuals) were studied from the Wanzhou District who were diagnosed with RT-PCR-confirmed SARS-CoV-2 infections but without relevant clinical symptoms in the "preceding 14 days and during hospitalization". The mean duration of viral shedding in the asymptomatic group was 19 days with an inter-quartile range of 15-26 days. The asymptomatic group had a significantly longer duration of viral shedding than the symptomatic group (log range $P=$ 
0.028). Data including: "asymptomatic individuals exhibited lower levels of 18 pro- and antiinflammatory cytokines".

A report in the journal "Patient" on March 31, 2020 described asymptomatic and mild COVID-19 ${ }^{20}$. Dr. Mary Lowth provocatively inquires about the meaning of mild, moderate, severe, and critical cases. She states: "There are, as yet, no clear guides for patients to tell them what doctors mean by mild, moderate or severe COVID-19" and affirms that "Some guidance on classifying illness is appearing in research papers and epidemiological reports, but it's not very specific. For example, the broad definition of moderate disease seems to be that it's worse than mild disease but not severe." Five terms used to describe levels of illness: asymptomatic, mild, moderate, severe, critical are said to be consistent with published descriptions so far. The author states:

Each level is considerably less common that the previous one... When formal, agreed guidance on what we call mild, moderate and severe cases is published these may differ slightly... Most people who are young and healthy are likely to be in the group one or two (asymptomatic or mild). However there are reports emerging that some young healthy people have become very unwell and at least one in the UK has died. In percentage terms this appears to be very low, but the chance is not zero... you have a chance of moving further down the list into moderate, severe and critical infections if you are over 60 (and an even higher chance if you are over 75); your health is normally compromised by heart disease, lung disease, cancer and chemotherapy, immune deficiency; you have a solid organ transplant... even in the over-80s group, very significantly more people will have mild or moderate disease than severe disease... You may well not fit any one classification exactly. You may be between two. You may move from mild to moderate symptoms, and then back to mild. You may have symptoms from more than one group. Illness is like that.

The difficulty in ascribing symptoms is shown by the following statement made by this author:

Being asymptomatic means you have no symptoms. However, there are reports of loss of sense of smell in asymptomatic people. Technically even that is a symptom. Patients with mild illness have flu-like symptoms. These may include dry cough and mild fever, but the fever may not reach $37.8^{\circ} \mathrm{C}$, and there may sometimes be little or even no cough. Patients might notice a feeling of being a bit more breathless than normal on exercise, but they are not out of breath on normal household activity... It is possible that a large proportion of the population will have COVID-19 yet have no symptoms at all, but we don't know that yet.

The degree to which this describes the actual situation for doctors and patients alike, documents the need to address asymptomatics in patient care and in societal policies. A study was focused on the fact that asymptomatic cases are "difficult to be effectively identified and therefore pose considerable challenges in the containment of the disease and prevention of new outbreaks" ${ }^{21}$. A total of 511 confirmed cases of COVID-19 included 100 asymptomatic (at the time of testing) were enrolled in the study from January 25 to February 20, 2020. They came from hospitals in 21 cities and 47 counties or districts in Sichuan Province. Epidemiological and clinical characteristics were compared. Asymptomatic 
patients were younger, compared to asymptomatics $(P<0.001)$, had similar co-morbidity percentages ( $P$ $=0.609)$, and came from higher altitude areas with lower population mobility $(P<0.001)$ with better defined epidemiological history $(P<0.001)$. Of well-documented asymptomatic cases, $27.4 \%$ developed delayed symptoms after pathogenic diagnosis. Of the symptomatic cases, $60 \%$ demonstrated evidence of pneumonia on the initial CT, including what were described as "well-recognized features of coronavirus disease-19. None of the asymptomatic individual died. Two elderly individuals with initially asymptomatic infection developed severe symptoms during hospitalization."

\section{RELEVANT STUDIES}

\section{Iceland Retrospective Study}

In an Iceland study of 13,080 who volunteered for screening, $100(0.8 \%)$ tested positive (remaining stable over the course of 20 days) for SARS-COV-2; all were aged 10 years or older; none of 848 children younger than 10 years tested positive ${ }^{22}$. Among all with a positive test, $43 \%$ had no symptoms of COVID-19 at the time of testing. However, the authors stated "symptoms almost certainly developed later in some of them". The significance of these data is questionable. However, the data show the slowness of the spread of the virus in this population. The authors suggest slow-spreading was because of self-isolation, quarantining, and social distancing. Relevant to our review, the authors asked participants who had respiratory symptoms, which they described as more than mild, not to participate in population screening; close to half the participants reported symptoms, most commonly rhinorrhea and coughing. Thus, a weakness in the design of the study was that persons who were concerned about potential infection may have been more likely than others to participate.

\section{An Outbreak in Italy with Statistical Analysis}

The results of a study from the municipality of Vo' Italy had 36 authors, was published in Nature by an accelerated process, and included statistical analysis ${ }^{23}$. On February 21, 2020 a resident of Vo' died of pneumonia due to SARS-CoV-2 infection. It was the first COVID-19 death in Italy and the "whole municipality was locked down for 14 days." The prevalence of asymptomatic infections was determined from two surveys. One survey was conducted "around the time the lockdown started" and a second survey was done at the "end of the lockdown" (14 days later). Nasopharyngeal swabs were collected and tested from 2,812 and 2,343 subject ( $85.9 \%$ and $71.5 \%$ of the eligible study populations) at the beginning and ending of the lockdown. In the first survey, the prevalence of infection was $2.6 \%(95 \%$ confidence interval of 2.1-3.3\%). The prevalence of infection in the second survey was $1.2 \%$ (95\% confidence interval of $0.8-1.8 \%)$. The authors stated:

Notably, $42.5 \%$ (95\% confidence interval $31.5-54.6 \%$ ) of the confirmed SARS-CoV-2 infections detected across the two surveys were asymptomatic (i. e. did not have symptoms at the time of swab testing and did not develop symptoms afterwards). The mean serial interval was 7.2 days (95\% confidence interval 5.9-9.6\%). We found no statistically significant differences in the viral loads of symptomatic versus asymptomatic infections (p-values 0.62 and 0.74 for $E$ and $R d R p$ genes, respectively, Exact Wilcoxon-Mann-Whitney test). 
A report in May of $2020^{24}$ stated that there were limited data available for asymptomatic infections and it presented the clinical characteristics of 24 cases with asymptomatic infection in Nanjing, China in clinic and in the community from January 28 to February 9, 2020. None of the asymptomatic cases "presented any obvious symptoms while (sic) nucleic acid screening." Five cases $(20.8 \%)$ developed symptoms (fever, cough, fatigue, etc.) during hospitalization. Twelve (50\%) cases showed typical CT images of ground-glass chest and 5 (20.8\%) presented stripe shadowing in the lungs. The remaining $7(29.2 \%)$ cases showed normal $\mathrm{CT}$ images and had no symptoms during hospitalization. These 7 cases were younger (median age: 14.0 years; $P=0.012$ ) than the rest. None of the 24 cases developed severe COVID-19 pneumonia or died. The median communicable period, defined as the interval from the first day of positive nucleic acid tests to the first day of continuous negative tests, was 9.5 days (up to 21 days among the 24 asymptomatic cases.). "Overall, the asymptomatic carriers identified from close contacts were prone to be mildly ill during hospitalization. However, the communicable period could be up to three weeks and communicated patients could develop severe illness." A table, very useful for the asymptomatic issue of this review, detailed the data for date of diagnosis, date of tests, and symptom presentation.

A review article (available online March 4, 2020) addressed "facts and myths" regarding the asymptomatic carrier state of SARS-CoV- ${ }^{25}$. It was stated that, at the time the article was written, there were more than 75,000 cases reported from 32 countries/regions and more than 2,000 deaths worldwide. The authors stated:

The COVID-19 can present as an asymptomatic carrier state, acute respiratory disease, and pneumonia... Severe cases are more likely to be older patients with underling co-morbidities compared to mild cases... The clinical manifestations of COVID-19 are protean, which can include asymptomatic carrier, ARD, and pneumonia of varying degrees of severity. First, asymptomatic cases were diagnosed based on positive viral nucleic acid test results, but without any COVID-19 symptoms, such as fever, gastrointestinal, or respiratory symptoms, and no significant abnormalities on chest radiograph [2 references cited]. However, the transmission of COVID-19 through asymptomatic carriers via person-to-person contact was observed in many reports [4 references cited].

Usefully, a chronology of countries with cases at this early period of the outbreak was recorded and epidemiology, age and sex, risk factors, incubation period, clinical manifestations, treatment, and outcomes were addressed. As outcomes, the overall mortality rate was stated to be $2.9 \%$ [reference cited]. Initial studies reported that the mortality rate associated with SARS-CoV-2 pneumonia ranged from $11 \%$ to $15 \%$ [reference cited].

Most relevant to the current review, there was a section titled "Uncertain Issues" and it was stated that:

The clinical manifestation of COVID-19 ranges from the asymptomatic carrier state to severe pneumonia; however, most early reports only showed the findings of SARS-CoV-2 pneumonia, in which the ratio of male patients was much larger than that of female patient, there were no pediatric cases, and the mortality rate was high [3 references cited]... most studies, especially 
those with a large patient population, were conducted in China, and the study of asymptomatic carriers was limited. More studies are needed to clarify the epidemiologic characteristics of COVID-19 ... viral load detected in asymptomatic patients was similar to that found in symptomatic patients; however, the viral loads from patients with severe diseases were higher than those in patients with mild-to-moderate presentations.

All confirmed cases of COVID-19 in Hunan Provincial People's Hospital in Changsha, China between January 22 and February 15, 2020 were used in a study ${ }^{26}$. Cases were detected by real-time reverse transcription polymerase chain reaction assay. There were 24 confirmed cases in the fever outpatient department of the hospital; 3 were asymptomatic; 3 had mild disease; and 3 had moderate disease. It was concluded that COVID-19 can be asymptomatic or exhibit mild disease. The main objective of the study was concern for spread of the disease and not the quantification of asymptomatic cases.

A report of asymptomatic carriers and pre-symptomatic cases studied 71 patients who were hospitalized for treatment or isolation ${ }^{27}$. It was stated:

Patients who had any of the following features at quarantine release were analyzed: (1) asymptomatic carrier $(n=10)$, i.e. those who had no symptoms in the 14 days preceding diagnosis until release from quarantine; $(2)$ incubation period patients $(n=3)$, i. e., those who were asymptomatic during the 14 days preceding diagnosis, but became symptomatic during quarantine. The median age of the asymptomatic carriers was 31 years (inter-quartile range 17.8-55.8 years). Three patients who were asymptomatic on admission developed myalgia, fever, and a cough 1 or 2 days afterward, without pneumonia. In two of the three patients, the viral load during the incubation period was very high ( $\mathrm{Ct}$ value $<20$ ), but all patients first reached $\mathrm{C} t>35$ by day 14 . In the asymptomatic SARS-CoV2 carriers, the viral load on admission was not relatively high. The median time to first RT-PCR Ct $>35$ result after diagnosis in the asymptomatic carriers was 4.5 days (range 2.0-8.5 days) and all asymptomatic cases first reached a $\mathrm{Ct}>35$ within 14 days after diagnosis... RT-PCR was intermediate or negative 14 days after diagnosis in entirely asymptomatic individuals who were not given any antiviral agents. Although the presence of viral RNA in specimens does not distinguish between infective and non-infective viruses, a study found that live virus could not be detected by culture in cases with $\mathrm{Ct}>35$ [reference cited]... patients had high viral loads before the onset of symptoms... In summary, patients who have COVID-19 may already be infectious when there are no symptoms, and 14 days of isolation may be sufficient in entirely asymptomatic cases.

Asymptomatic transmission to children was reported in a familial cluster infection ${ }^{28}$. Two children were admitted on February 1, 2020, with mild symptoms, to an isolation ward to a hospital in China with the diagnosis of COVID-19. The evidence indicated they acquired COVID-19 from their mother who did not develop symptoms until February 6, 2020 with an asymptomatic period of 15 days. The mother became nucleic acid positive "until February 21, 2020, 15 days after symptoms onset, 30 days after her return from Wuhan." The primary focus of this report was documentation of transmission via asymptomatic "carriers". 
Epidemiological features of 36 Chinese children with COVID-19 were reported in an observational cohort study ${ }^{29}$. From January 17 to March 1, 20220, 36 children (mean age 8.3 [SD 3.5] years) were diagnosed with COVID-19. A Figure is presented that tracked the trajectory of the disease and its treatment in four pediatric patients with different symptoms manifestations, including asymptomatic. Nineteen (53\%) had moderate pneumonia; 17 (47\%) were described as "mild clinical type" and either were asymptomatic, $10(28 \%)$ or had actuate upper respiratory symptoms, 7 (19\%). On admission, fever was common, 13 (36\%), dry cough, 7 (19\%). Mean time in hospital was 14 (SD 3) days and by Feb 28, all patients were described as "cured".

\section{Quarantined Ship's Passengers}

Cruise ship outbreaks of COVID-19 offer unique opportunities for assessing spread of the infection according to the $\mathrm{CDC}^{30}$ which also stated that more than 800 cases (as of March 27, 2020) of COVID-19 occurred during outbreaks on three cruise ship voyages plus other cases linked to other cruises. Transmission occurred across multiple voyages from ship to ship by crew members and both crew and passengers were infected with 10 deaths.

\section{The Diamond Princess Cruise Ship}

The Diamond Princess cruise ship case is one of the more thoroughly investigated outbreaks and it will be described in detail. During February 2-23 of 2020, the largest cluster of cases outside mainland China occurred on the Diamond Princess ship subsequently quarantined in Yokohama, Japan ${ }^{30}$. There were 3,711 Diamond Princess passengers and crew; 712 (19.2\%) tested positive for SARS-CoV-2 and 331 (46.5\%) were asymptomatic when tested. The patients with symptoms were a total of $381 ; 37(9.7 \%)$ required intensive care, and there were $9(1.3 \%)$ fatalities. Infections also occurred among Japanese responders. When this report was prepared (March 13), 107 (25\%) of 428 U.S. passengers and crew had tested positive for COVID-19; 11 passengers (median age 75) were in hospital and 7 were described to be in serious condition. This CDC account ${ }^{30}$ further stated: “... the Diamond Princess demonstrated a high proportion (46.5\%) of asymptomatic infections at the time of testing.

A statistical model of this outbreak "suggests" that $17.9 \%$ of infected persons never develop symptoms

${ }^{31}$. This report is an example of the problem arising from many reports of COVID-19; scientists earnestly try to do the best they can with data that sometimes is collected other than as part of a scientific study. Indeed, a statistical approach was used to deal with the proportion of asymptomatic individuals calculated from the results accumulated in a table of test results for the virus ( $n=3,711$ individuals). In describing this report, I am compelled to note that I appreciate that this was not designed as an experiment. However, only limited conclusions can be scientifically drawn from the use of data that was collected in the course of sincere efforts to deal with the medical and other issues related to this tragic outbreak. In my view, conclusions about the proportion of asymptomatic cases are especially problematic.

Data was obtained over 16 days and placed in 9 categories in a detailed Table. Of 144 possible entries, 63 were noted to be not available (NA). Most relevant to this review, in the category "Number of asymptomatic cases" 10 of 16 entries (62.5\%) were NA. Of the 3,711 passengers and crew, 3,063 were 
tested at some point over the 16 days. How people were selected to be tested and when they were tested was not clearly stated; what bias did this introduce? The tests included 2 NA, a daily low of 6 and a high of 681 , with more tests later in the test days. There were 634 positive tests, and 320 asymptomatic cases. By definition an asymptomatic case is a person without symptoms who tests positive. In this study such a person cannot be identified until tested. Obviously, everyone was not tested each day, so the number of people with no symptoms who test positive is not known; the number reported in this study (daily and cumulative number) is reduced in value. Collected on a daily basis, it is almost arbitrary because the number of people eligible to be so classified varies from NA, 6 tests minimum and 681 tests maximum (and this is among an irregularly decreasing number of tested people). These authors discussed the relevance of asymptomatic cases and stated:

...another key epidemiological parameter that could inform the intensity and range of social distancing strategies to combat COVID-19 is the asymptomatic proportion, which is broadly defined as the proportion of asymptomatic infections among all the infections of the disease. Indeed, the asymptomatic proportion is a useful quantity to gauge the true burden of the disease and better interpret estimates of the transmission potential. This proportion varies widely across infectious diseases, ranging from $8 \%$ for measles and $32 \%$ for norovirus infections up to $90-95 \%$ for polio [a reference is cited]... there is accumulating evidence indicating that a substantial fraction of SARS-CoV-2 infected individuals are asymptomatic [3 references cited].

To assess the asymptomatic data, these authors stated: “... estimation of the asymptomatic proportion needs to be handled carefully since real-time outbreak data are influenced by the phenomenon of right censoring." They chose to do statistical modeling analyses and the reported asymptomatic proportion was $17.9 \%$ with a $95 \% \mathrm{Crl}$ of 15.5 to $20.2 \%$. The data, before this statistical analysis that assumed right censoring, was as follows. There were 634 confirmed cases with 306 symptomatic and 328 asymptomatic. They stated: “The proportion of asymptomatic individuals appears to be $16.1 \%(35 / 218)$ before 13 February, 25.6\% (73/285 on 15 February, 31.2\% (111/355) on 16 February, 39.9\% (181/454) on 17 February, 45.4\% (246/542) on 18 February, 50.6\% (314/621) on 19 February and 50.5\% (320/634) on 20 February." The authors decided to do statistical modeling to assess this data. They stated: "The reported asymptomatic cases consist of both true asymptomatic infections and cases who had not yet developed symptoms at the time of data collection but became symptomatic later, i.e. the data are right censored." The authors describe the statistical methods and equation used, and concluded: "Our estimated asymptomatic proportion is at $17.9 \%$..." They described the study as "not free of limitations... laboratory tests by PCR were... focusing on symptomatic cases... if asymptomatic cases were missed... we have underestimated the asymptomatic proportion." Other limitations also were appropriately described.

These authors are to be affirmed for their efforts to assess asymptomatic cases and the correctness of their statistical approach and the number reported will be resolved by future study.

A report in the New England Journal of Medicine ${ }^{32}$ describes the "natural history" of asymptomatic SARS-CoV-2 infection in part of the cohort from the Diamond Princess passengers and crew. A total of 96 persons infected withSARS-CoV-2 who were asymptomatic at the time of testing and 32 "cabin mates" 
who tested negative on the ship were transferred to a hospital in central Japan between February 19 and February 26, 2020 for continued observation. Clinical signs and symptoms of Covid-19 subsequently developed in 11 of the 96 after a median of 4 days (inter-quartile range, 3 to 5; range 3 to 7) after the first positive polymerase chain-reaction (PCR) test. The authors concluded this meant that they were pre-symptomatic rather than asymptomatic. The risk of being pre-symptomatic increased with increasing age. The odds ratio for being asymptomatic with each 1-year increase in age was 1.08 (confidence interval 1.01 to 1.16). Eight of 32 cabin mates with a negative PCR test on the ship had a positive PCR within 72 hours after arrival in the hospital but remained asymptomatic.

\section{Unnamed Cruise Ship}

An account was described to be the first complete COVID-19 testing of all passengers and crew on an isolated cruise ship during the current COVID-19 pandemic ${ }^{33}$. The ship was not named in the account, but its route was similar to that taken by British explorer Ernest Shackleton in 1915-1917. From departure in mid-March 2020 for 28 days the ship had no outside human contact. A remarkable $81 \%$ were asymptomatic among 128 with positive tests for infection by the virus. The report states:

Of the 217 passengers and crew on board, 128 tested positive for COVID-19 on reverse transcription-PCR (59\%). Of the COVID-19- positive patients, $19 \%$ (24) were symptomatic; $6.2 \%$ (8) requited medical evacuation; $3.1 \%$ (4) were intubated and ventilated; and the mortality was $0.8 \% \ldots$ The majority of COVID-19-positive patients were asymptomatic $(81 \%, 104$ patients)... despite 128 (59\%) of the population testing positive, fever and mild symptoms were present in only 16 of 128 CoVID-19-positive patients (12.5\%).

\section{The Aircraft Carrier Theodore Roosevelt}

The Aircraft Carrier U.S.S. Theodore Roosevelt had an outbreak of COVID-19 and a report was posted on MMWR on June 9, 2020 as early release (https://www.cdc.gov/mmwr). A full account was provided in the publication reviewed here ${ }^{34}$. In mid-January, the USS Theodore Roosevelt was deployed to the western Pacific. An outbreak of COVID-19 occurred and the ship stopped at Guam near the end of March. Approximately 1,000 service members were said to have been infected with SARS-CoV-2 and the U.S. Navy and the CDC investigated the outbreak during the period April 20-24. Service members (total 382) volunteered to complete to complete questionnaires and provide serum samples which were described as "... a convenience sample comprising $27 \%$ of 1,417 service members staying at the base on Guam or on the ship." The 1,417 were said to include: "... persons who were previously infected, currently infected, or never infected. Among the 382 service members, 267 (70\%) also provided a nasopharyngeal swab specimen."

This report also stated:

Serum specimens were tested for antibody reactivity using a CDC-developed, SARS-CoV-2 spike protein enzyme-linked immunoabsorbent assay (ELISA) (a pan-immunoglobulin assay) as an indicator of previous SARS-CoV-2 exposure and infection; signal threshold ratio equal to or greater than 1 was defined as a positive ELISA result. ELISA-positive specimens were further 
tested for neutralizing antibodies using a micro-neutralization assay to detect presence of SARSCo-V-2 inhibiting antibodies (antibody titers greater than 40 defined as positive)... Real-time reverse-transcriptase-polymerase chain reaction... was used to detect SARS-CoV-2 RNA. Previous or current SARS-CoV-2 infection was defined as a positive real-time RT-PCR result or positive ELISA result.

Among 382 survey participants, 289 (75.7\%) were male; their median age was 30 years (inter-quartile range of 24-35 years). Among 238 (62.0\%) of participants with previous or current SARS-CoV-2 infection, 194 (81.5\%) reported one or more symptoms; 44 (18.5\%) were asymptomatic; two (0.8\%) were hospitalized for COVID-19. Among 284 symptomatic participants, $68.3 \%$ with previous or current SARSCoV-2 infections and $31.7 \%$ without, loss of taste (ageusia) or smell (anosmia) were the symptoms most strongly associated with previous or current infection (odds ratio $=10.3$ ), followed by fever (odds ratio = 2.8 ), chills (odds ratio $=2.7$, and Myalgia (odds ratio $=2.6$ )

The authors defined symptoms as specified by the Council of State and Territorial Epidemiologists (CSTE) for COVID-19. The following description was provided: “... category A (at least cough or shortness of breath/difficulty breathing) and category B (no cough or shortness of breath, but two or more other symptoms*) or neither." The * was defined as: "Fever, chills, muscle pain, headache, sore throat, new taste or smell disorder." Interpretation of "or neither" is problematic.

Asymptomatic cases can be defined in myriad ways and therein is the potential for great variability. This report tabulates a large amount of data. The issue addressed that is relevant to this review, the proportion of cases that are asymptomatic is obviously very different depending on how asymptomatic is defined. The data table tabulates "Current or previous SARS-CoV-2 infections* ( $N=238$ )" with * defined as "Current or previous SARS-CoV-2 infection is defined as a positive RT-PCR test result or a reactive antibody result by testing performed at CDC laboratories on specimens collected during April 20-24, 2020." The data is tabulated as "No. (\%)" but because disparate data is listed, there is confusion and I could not verify some percentages. However, it is significant that 16 different symptoms are listed and 5 reach "unadjusted" odds ratios for "Infection versus no infection $(95 \% \mathrm{Cl})$ " with a P-value " $<0.05$ considered statistically significant". I found it meaningful that they included a category "Sought medical care for symptoms" and the values were: $59.3 \%$ for "Current or previous SARS-CoV-2 infection*" $(\mathrm{N}=238)$ [Note that * was previously defined.], and 38.9\% for "No. evidence of SARS-CoV-2 infection ( $\mathrm{N}=$ 144)". No reason was given for what appears to be a high reporting of 16 different "Individual symptoms" among the non-infected. It also is to be noted that for the category "Hospitalized", the numbers 2 and 0 , respectively are given for those with infections vs. those without. Almost any conclusion can be drawn about the frequency of occurrence of asymptomatic cases. In the authors' defense, they did not claim to focus on the issue of asymptomatic cases. I draw attention to this lost opportunity (which is understandable).

\section{The Aircraft Carrier Charles de Gaulle}

The outbreak that occurred on the Charles-de-Gaulle aircraft carrier has been widely covered in the press but also collected in one account ${ }^{35}$; however, I did not find any article published in a scientific 
journal. This limits the usefulness of this outbreak which otherwise provides information of potential value for the issue of the proportion of cases that are asymptomatic. This outbreak is included here because it exemplifies the status of data bearing on asymptomatic COVID-19. It is reported that nearly $2 / 3$ of the cases tested positive when the ship returned to France in mid-April. The data appears to be 1046 out of 1760 (or 1767). I cite one source ${ }^{35}$ because the accounts appear in an evolving fashion, may be unreliable regarding facts, and there was a failure to provide necessary context.

\section{Repatriated Citizens}

The following data was extracted from a brief, Rapid Communication ${ }^{36}$. Note the dates which are relevant to the issue of right censoring which appears in other reported analyses. On March 20, 2020, repatriates from the UK provided the following data: number of passengers repatriated $=357$; number with symptoms $=0$; number that tested positive $=13$; as of April 6, 2020, the number which were symptomatic $=10$ (therefore the number asymptomatic $=3$ ) and 1 of these tested positive. If my extractions of the data are correct (the entries are incomplete and briefly defined), a conclusion of right censoring of the numbers as of April 6 might be considered. This is possible because some or all of the passengers who had no symptoms on March 20 (most specifically some or all of the 13 who tested positive) could have been pre-symptomatic.

On February 3, 2020, Fifty-six asymptomatic Italian citizens were medically evacuated from Wuhan, China to Rome, Italy and quarantined for 14 days in a Cecchignola military complex ${ }^{37}$. Before leaving Wuhan, all subjects were afebrile and otherwise asymptomatic. On February 5 , all were screened for SARS-Co-V-2 RNA using real-time RT-PCR on nasal swabs. Only one swab was positive (for both the envelope protein $E$ and RNA-dependent RNA polymerase gene (RdRp). This individual was described as paucisymptomatic (having few symptoms).

The authors stated:

Our patient was tested for SARS-CoV-2 within the WHO contact protocol applied to the 56 Italians... Otherwise, with no clear exposure or direct contact with possible sources of SARS-CoV2 infection, he would not have been included in the COVID-19 case definition and not considered for viral screening... in our case, the patient, still undiagnosed, did probably not transmit the SARS-CoV- 2 infection to his asymptomatic Chinese close contacts and to the other 55 Italian citizens... Further studies are needed to better understand he clinical spectrum of COVID-19 at hospital and community levels, the role of pauci-/asymptomatic subjects in viral transmission and the clinical relevance of viral persistence in non-respiratory samples as potential sources of Sars-CoV-2.

A report ${ }^{38}$ was focused on estimating the effectiveness of symptom risk screening to prevent the spread of COVID-19 and included relevant findings for asymptomatic cases. Because of the nature of the wording of this publication it must be quoted at length:

Even under best-case assumptions, we estimate that screening will miss more than half of infected people... we find that most cases missed by screening are fundamentally undetectable, 
because they have not yet developed symptoms and are unaware they were exposed... screening has become a ubiquitous tool... to contain local spread of COVID-19... It is widely recognized that screening is an imperfect barrier to spread... due to the absence of detectable symptoms during the incubation period; variation in the severity and detectability of symptoms once the disease begins to progress; imperfect performance of screening equipment or personnel; or active evasion of screening by travellers [preferred spelling in U.K.]... Previously we estimated the effectiveness of traveller screening for a range of pathogens that have emerged in the past, and found that arrival screening would miss $50-75 \%$ of infected cases even under optimistic assumptions [reference cited]... We define subclinical cases as those too mild to show symptoms detectable in screening (fever or cough) after passing through the incubation period (i.e. once any symptoms have manifested). The true fraction of subclinical Covid-19 cases remains unknown... many lab-confirmed COVID-19 cases have not shown detectable symptoms on diagnosis [3 references cited]. About $80 \%$ of clinically attended cases have been mild [reference cited], and clinically attended cases have been conspicuously rare in children and teens [4 references cited].

Data was placed in a table that included the categories parameter values, accompanying uncertainties, and assumptions. Parameters included: mean incubation period, incubation period distribution, percent of cases subclinical (no fever or cough), $R_{0}$, percent of travelers aware of exposure risk, sensitivity of infrared thermal scanners for fever, probability that travelers self-report exposure risk, and time from symptomatic onset to patient isolation (after which we assume travel is not possible). Individual outcome probabilities were shown based on percentage of exposed individuals detected or missed vs. days since exposure. The most germane data for this review are for the percent of subclinical cases (no fever or cough) and estimates were provide as: best case scenario $=5 \%$; middle case scenario $=25 \%$; worst case scenario $=50 \%$. References are cited for the clinical data: fever, cough, and asymptomatic at diagnosis as follows: $31.2 \%, 62.2 \%$, and $70.0 \%$, respectively.

This report is among the most thorough published to my knowledge; however, it was not specifically focused on the issue of asymptomatics. The data were designed for assessing the effectiveness of symptom screening for preventing spread of COVID-19. They also reveal the complexity of assessing the complex term "asymptomatic" in the context of this disease. All of the discussed factors are relevant for assessing the consequences of asymptomatic cases for determining the prevalence of COVID-19.

\section{Homeless Shelters}

Tests at a homeless shelter in Boston provided interesting results ${ }^{39}$. However, the population (homeless) was not representative of the population at large. An increasing number of COVID-19 cases from a large homeless shelter prompted SARS-CoV-2 testing what was stated to be all of the remaining residents (408 people). They were tested on two days (April 2 and 3) for symptoms and PCR tested for virus. The authors stated that a total of 147 (36.0 \%) were PCR-positive and $361(88.5 \%)$ had no symptoms. The breakdown of symptoms for SARS-CoV-2 PCR-positive and PCR-negative people was provided in a data table. 
A described limitation of the study was stated to include "... the cross-sectional nature of the study at a single shelter in Boston where several symptomatic individuals had been removed through prior symptoms screening or self-referrals to outside care." Sixteen were diagnosed with COVID-19 prior to April 2, and 6 concurrently diagnosed at outside facilities on April 2 or 3 (3.92\% and 1.5\%, respectively, of the number in the test, 408).

A cluster of cases at a Los Angeles homeless shelter was discovered when a COVID-19 case was diagnosed at a homeless shelter in downtown Los Angeles. Results were reported ${ }^{40}$ but I have not found a peer-reviewed publication. The first case was found on March 28, 20202. Tests were done on 178 people as of April $21 ; 43$ were positive (37\%) and $63 \%$ of these cases were asymptomatic. The shelter was said to have had 1000 people "at one time", and "they are now down to just about 400 people... with 184 people moved over the past two weeks". An update on April 22 stated that $43(24.2 \%$ of 178 tests) were positive and 27 (63.8\%) of these were asymptomatic. These data are not adequate for scientific analysis. However, there appears to be little or no basis to conclude that this population would have improved resistance, practiced social distancing, wore masks, had improved diets, or had reduced co-morbidities. It might be argued that exposure to sunlight could raise vitamin D levels and low vitamin $\mathrm{D}$ has been speculated to be possibly detrimental for COVID-19.

\section{Obstetric Patients}

An account was said to report universal screening of obstetric patients ${ }^{41}$. Between March 22 and April 4, 2020, women who delivered babies at 2 New York City hospitals were tested for SARS-CoV-2. Among 214 who were tested, 33 (15.4\%) were positive and 29 (87.9\%) were asymptomatic. It was noted that subsequently "fever developed in $3(10 \%)$ before postpartum discharge (median length of stay, 2 days)"; however, two of these with fever were treated with antibiotics because they were thought to have endomyometritis.

\section{Healthcare Workers}

During the 3 week period in 2020 (April 6-24), 1032 asymptomatic health care workers were screened for SARS-CoV-2 in a large United Kingdom teaching Hospital, and 3\% were positive ${ }^{42}$. Of these, $57 \%$ were described as "truly asymptomatic/pauci-symptomatic" and $40 \%$ "had experienced symptoms compatible with coronavirus disease 2019 (COVID-19) > 7 days prior to testing, most self-isolating, returning well." Symptomatic staff and symptomatic household contacts were also tested. The clinical presentation of COVID-19 was described as: "can include minimal or no symptoms." Asymptomatic and or pre-symptomatic transmission is clearly reported and is estimated to account for around half of all cases and a reference is cited. The testing procedure was carefully done and $5 \%$ were positive. A table provides the clinical criteria of major (three) and minor (ten) symptoms used for "estimating pre-test probability of COVID-19". Clinical vignettes were used to assist in describing the following categories: completely asymptomatic, pre-symptomatic, low clinical probability of COVID, medium clinical probability of COVID, high clinical probability of COVID. This clearly documents the difficulty of categorizing individuals as "asymptomatic" and is the basis for the caution used in this review for assessing all studies addressing this issue. The focus of this paper clearly was on the effect of 
asymptomatic carrier among healthcare workers, and its effect on transmission of COVID-19. Relevant data and conclusions included:

$12 / 30(40 \%)$ individuals from the HCW (health care workers) asymptomatic screening group reported symptoms $>7$ days prior to testing, and the majority experiencing symptoms consistent with a high probability of COVID-19 had appropriately self-isolated during that period. Patients with COVID-19 can remain SARS-CoV-2 PCR positive for a median of 20 days (IQR 17-24) after symptom onset" [a reference is provided]... Our data clearly demonstrate that focusing solely on the testing of individuals fitting a strict clinical case definition for COVID-19 will inevitably miss asymptomatic and pauci-symptomatic disease.

\section{Nursing Facilities}

An article was unusually thorough in reporting SARS-CoV-2 at a nursing facility ${ }^{43}$. The introduction to this account states that the first reported case of COVID-19 in the United States was diagnosed in a resident of Snohomish County, Washington on January 20, 2020. In late February, an outbreak was identified in a nursing facility in nearby King County. Subsequently, another outbreak in a different nursing facility also in King County occurred and it was the source of the information presented. The Centers for Disease and Prevention (CDC), the Public Health- Seattle and King County investigated.

Two surveys, described as "serial point-prevalence", were done 1 week apart. Symptoms present during the preceding 14 days were recorded. Asymptomatic residents who tested positive were reassessed 7 days later. Those who tested positive were categorized as symptomatic with typical symptoms (fever, cough, or shortness of breath), symptomatic with only atypical symptoms, pre-symptomatic, or asymptomatic. Twenty-three days after the first positive test, 57 of 89 residents (64\%) tested positive for SARS-CoV-2. Among 76 residents who participated in point-prevalence surveys, 48 (63\%) tested positive. Of these 48 residents, 27 (56\%) were asymptomatic at the time of testing; 24 subsequently developed symptoms (medium time to onset, 4 days). These 24 residents were properly labeled as presymptomatic and they had a median rRT-PCR cycle threshold value of 23.1, and viable virus was recovered from 17. As of April 3, of the 57 residents with SARS-CoV-2 infections, 11 had been hospitalized ( 3 in intensive care) 15 had died (mortality $26 \%$ ). Of the 34 residents whose specimens were sequenced, 27 (79\%) fit into two clusters with a difference of a single nucleotide. The authors concluded (in part): "More than half of residents with positive test results were asymptomatic at the time of testing and most likely contributed to transmission." The actual data for participants in point-surveys was $56 \%$ asymptomatic when first tested; however, $50 \%$ of these subsequently became symptomatic. Therefore, half of the non-symptomatic should be labeled pre-symptomatic (with a median time of 4 days). This provides some reasonably accurate data for assessing the issue of right-censoring that has been presumed in other studies.

This report addresses the issue of pre-symptomatic and symptomatic cases with data gathered over a period of time and, therefore, it provides information about the issue of whether patients might not have symptoms when first tested and found to be positive, but who might later develop symptoms. In most reports, information is lacking about whether the category of pre-symptomatic cases are 
significant. This report, however, was focused primarily on the issue of asymptomatic cases as they relate to transmission. There was detailed information about the health status of the individuals. Ninetyeight $\%$ of the SARS-CoV- 2 positive individuals (48), and $100 \%$ of the negative individuals had some coexisting health condition. The most prevalent condition was cardiovascular disease with $81 \%$ for residents who tested positive and $61 \%$ for those testing negative, and the amounts of 7 other co-existing conditions were specified. The mean ages for those testing positive were $78.6 \pm 9.5$ years and $73.8 \pm 11.5$ for those testing negative. Obviously, any conclusions about this outbreak are not appropriate for total populations.

Another outbreak occurred in King County, Washington in a long-term care skilled nursing facility ${ }^{44}$. On March 1, 2020, a health care provider had a positive test result for SARS-CoV-2 after working while asymptomatic on February 26 and 28. By March 6 , seven residents of this facility were symptomatic and had positive test results for SARS-Co-2. On March 13, CDC performed symptom assessment and SARSCoV-2 testing for 76 (93\%) of the 82 residents. Residents were categorized as asymptomatic or symptomatic at the time of testing, based on the absence or presence of fever, cough, shortness of breath, or "other symptoms" on the day of testing or during the preceding 14 days. Among 23 (30\%) residents with positive tests results, $10(43 \%)$ had symptoms on the date of testing, and $13(57 \%)$ were asymptomatic. Seven days after testing, 10 of these 13 previously asymptomatic residents had developed symptoms and were categorized as presymptomatic at the time of testing. This affirms that designating a person in the category "asymptomatic" clearly can be dependent on when testing is done.

\section{Prisons}

In Ohio, at the Marion Correctional Institution, 2,300 inmates were tested out of a population said to be 2,500; 2028 tested positive but approximately $95 \%$ had no symptoms ${ }^{45}$. This U.S. News report states that in four state prison systems (Arkansas, North Carolina, Ohio, and Virginia) $96 \%$ of 3,277 inmates who tested positive for COVID-19 were asymptomatic (said to be 4693 total tests that included assessment of symptoms). This article states:

Some people diagnosed as asymptomatic when tested for the coronavirus, however, may go on to develop symptoms later, according to researchers... Ohio... has 49,000 prisoners in 28 facilities. A total 3,837 inmates tested positive for the coronavirus in 15 of those facilities. But the state has not yet provided results on symptoms for 1809 of them and did not identify the total number of tests conducted... Reuters surveyed all 50 state prison systems. Of the 30 that responded, most are only testing inmates who show symptoms, suggesting they could be vastly undercounting the number of infected... Florida and Texas... report a combined total of just 931 cases ... New York, the epicenter of the U.S. outbreak, has reported 269 positive cases among 51,000 inmates. All three states are testing only symptomatic prisoners... Tennessee... has tested 3,503 prisoners at Bledsoe... 651 were positive and most of them were asymptomatic." In Goldsboro, North Carolina all 723 prisoners were tested within one week. Of the 444 who were infected by the virus, $98 \%$ were asymptomatic. 
One inmate died at the prison. In what was described as mass testing at two Arkansas prisons, 751 inmates were infected and "almost all of them asymptomatic". The account did not state the total number of inmates who were tested. Michigan's Lakeland Correctional facility was said to houses some of the state's oldest and most medically frail prisoners. As of April 23, nine Lakeland inmates reportedly had died from COVID-19. This was said to be a third of the deaths across Michigan's 29 state prisons. The account stated that nearly half of Lakeland's 1,400 prisoners suffer from chronic underlying health conditions and that many are in wheel chairs. The account stated: "Of the 971 tested so far, 642, or about $66 \%$ were positive." A state official reportedly declined to disclose how many were asymptomatic but said that in the seven state prison system conducting mass tests, 49 inmates have died.

\section{Mission District in San Francisco}

A nonscientific account dated May 28, 2020 reported results of testing for COVID-19 ("in a four-day blitz at the end of April") in a 16 square blocks area in San Francisco known as Census Tract $229.01^{46}$. The report states: "About $2 \%$ of the people tested positive for the coronavirus... $53 \%$ of those who tested positive were asymptomatic."

\section{Clinical Manifestations in Children}

A review ${ }^{47}$ described the clinical, laboratorial, and radiological characteristics of children with COVID-19. The Medline database was searched between December 1, 2019 and April 6, 2020; inclusion criteria were defined and a total of 38 studies (1124 cases) were included. The severity was classified in 1117 cases: $14.2 \%$ was asymptomatic, $36.3 \%$ was mild, $46.0 \%$ was moderate, $2.1 \%$ was severe, and $1.2 \%$ was critical. The most prevalent symptom was reported to be fever (47.5\%) followed by cough (41.5\%), nasal symptoms (11.2\%), diarrhea (8.1\%), and nausea/vomiting (7.1\%). One hundred forty-five (36.9\%) children were diagnosed with pneumonia and 43 (10.9\%) upper airway infections were reported. Reduced lymphocyte count was reported in $12.9 \%$ of cases. Abnormalities in computed tomography were reported in $63.0 \%$ of cases. The most prevalent abnormalities reported were ground-glass opacities, patchy shadows, and consolidations. One death was reported. The authors concluded "Clinical manifestations of children with COVID-19 differ widely from adult cases. Fever and respiratory symptoms should not be considered a hallmark of COVID-19 in children."

A systematic review of image findings fromCOVID-19 in children was published May $18,2020{ }^{48}$. This review states: "Because children appear to be less severely affected than adults, their imaging appearances have not been extensively reported". Four databases were searched (Medline, Embase, Cochrane, Google Scholar) and 22 articles were included that reported chest imaging findings and associated outcomes. The authors concluded:

CT chest findings in children with COVID-19 are frequently normal or mild. Lower lobes are predominantly affected by patchy ground-glass opacification. Appearances at follow-up remain normal or improve in the majority of children. Chest $\mathrm{CT}$ imaging adds little to the further management of the patient and should be reserved for severe cases or for identifying alternative diagnoses. 
A case series, focused on early computed tomography findings in COVID-19 in pediatric patients in a single center in Zhongnan Hospital of Wuhan University from January 20, 2020 to February 28, 2020, was reported ${ }^{49}$. Results from four children were included. The report states: "All of them were asymptomatic throughout the disease course (ranging from 7 days to 15 days)..." It may be noted that this statement permits the definition of a disease with no symptoms. "Thin-section CT revealed abnormalities in three... one patient did not present with any... Unilateral lung involvement was observed in two patients, and one patient showed bilateral lung involvement... five small lesions were identified, including ground-glass opacity $(n=4)$ and consolidation $(n=1)$." The authors concluded: "Small patches of ground-glass opacity with sub-pleural distribution and unilateral lung involvement were common findings on CT scans of pediatric patients in the early stage of the disese."

\section{Quantification of Undetected Cases}

A study, reported April 10, $2020{ }^{50}$ was initiated at the National Institutes of Health in Bethesda to estimate the number of adults in the United States without a confirmed history of infection with SARSCoV-2 who have antibodies to the virus. It was designed as an observational, cross-sectional study, with a projected enrollment of 15,000, starting July 15, 2020 with a completion date of March 31, 2022. It includes a statement by Anthony S. Fauci, M.D., Director of NIAID:

This study will give us a clearer picture of the true magnitude of the COVID-19 pandemic in the United States by telling us how many people in different communities have been infected without knowing it, because they had a very mild, undocumented illness or did not access testing while they were sick.

The study was described as follows:

The presence of antibodies in the blood indicates a prior infection. In this 'serosurvey', researchers will collect and analyze blood samples from volunteers to provide critical data for epidemiological models. The results will help illuminate the extent to which the novel coronavirus has spread undetected in the United States and provide insights into which communities and populations are most affected.

The study reported:

To date, reporting of U.S. cases of COVID-19 has mostly relied on molecular tests that determine the presence of the virus in a person's airways using a noninvasive cotton swab. While these cotton swab-based tests rapidly and effectively identify active infection, they do not determine whether a person was previously infected with SARS-CoV-2 and recovered. Analysis will be done for IgG and IgM antibodies against SARS-CoV-2 and other tests done to evaluate the immune responses to the virus.

Matthew Memoli, principal investigator of the study and director of NIAID's Laboratory of Infectious Diseases Clinical Studies Unit said: “An antibody test is looking back into the immune system's history 
with a rearview mirror. By analyzing an individual's blood, we can determine if that person has encountered SARS-CoV-2 previously."

\section{A COMPARISON WITH FLU}

\section{The pandemic of 1957-1959}

For perspective, the global mortality impact of the 1957-1959 influenza pandemic as recently reported ${ }^{51}$ will be described. The report states:

Quantitative estimates of the global burden of the 1957 influenza pandemic are lacking. Here we fill the gap by modeling historical mortality statistics... The pandemic -associated excess respiratory mortality rate was $1.9 / 10,000$ population (95\% confidence interval, 1.2-2.6 cases/10,000 population) on average duration 1957-1959. Excess mortality varied 70-fold across countries; Europe and Latin America experienced the lowest and highest rates, respectively... Increases in the mortality rate relative to baseline were greatest in school-aged children and young adults, with no evidence that elderly population was spared from excess mortality... Overall, we attributed 1.1 million excess deaths (95\% confidence interval, 0.7 million - 1.5 million excess deaths) globally to the 1957-1959 pandemic.

The authors concluded:

The global mortality rate of the 1957-1959 influenza pandemic was moderate relative to that of the 1918 pandemic but was approximately 10 -fold greater than that of the 2009 pandemic. The impact of the pandemic on mortality was delayed in several countries, pointing to a window of opportunity for vaccination in a future pandemic.

\section{Most flu cases are asymptomatic}

A report in Lancet Respiratory Medicine in $2014^{52}$ assessed the effect of influenza on populations, including the "risk of infection, illness if infected, illness severity, and consultation rates". This was stated to be "essential to inform future control and prevention." Preseason and weekly illness reporting, and RT-PCR identification of influenza from nasal swabs were used to track the course of seasonal and pandemic influenza over five successive cohorts in England from 2006 to 2011 with 5,448 personseasons follow-up. On average, influenza infected $18 \%$ (95\% confidence interval 16-22\%) of unvaccinated people each winter. Of those infected, there were 69 respiratory illnesses (per 100 personinfluenza-seasons) compared with 44 respiratory illnesses (per 100 person-influenza-seasons) in those not infected with influenza. The age-adjusted attributable rate of illness of the infected was "23 illnesses per 100 person-seasons... suggesting most influenza infections are asymptomatic.... these figures did not differ significantly when comparing pandemic with seasonal influenza." For PCR-confirmed cases, those infected with the 2009 pandemic strain had "markedly less severe symptoms than those infected with seasonal H3N2." The authors' interpretation was: "Seasonal influenza and 2009 pandemic strain were characterized by similar high rates of mainly asymptomatic infection with most symptomatic cases self- 
managing without medical consultation. In the community the 2009 pandemic strain caused milder symptoms than seasonal H3N2."

A brief article provides additional perspective about the incidence of $\mathrm{flu}^{53}$. This article has several references which will not be included here because flu is not the focus of the review. However, it discusses six periods of influenza transmission between 2006 and 2011 in a "flu-watch study" that estimated the incidence of influenza infection, the proportion of infections that were symptomatic, and the proportion of symptomatic infections that resulted in consultation with a primary-care practitioner. It was stated:

The findings reaffirm earlier reports that there are high rates of serological evidence of influenza infection without corresponding disease... roughly $20 \%$ of the community shows serological evidence of influenza infection each season... most infections (about $75 \%$ ) are asymptomatic or at least so mild that they are not identified through weekly active surveillance for respiratory illness.

\section{FLU and COVID-19 Comparisons}

Belongia and Osterholm ${ }^{54}$ make the excellent point: "we are in uncharted waters" with the current threat of both COVID-19 and influenza. They then state: "Social distancing and stay-at-home orders are socially and economically disruptive, but can reduce demand on hospitals and protect vulnerable populations... [and] also reduce transmission of ... influenza..." They appear to accept cited numbers for COVID-19 deaths with the modest reservation that "... the death count is almost certainly higher".

Consider the evidence. As of June 10, 2020 based on CDC data for 20 States ${ }^{55}$ deaths from flu and pneumonia (as a percentage of population) were: $0.017 \pm 21 \%$, range $0.014-0.021$, median 0.017 , and lowest and highest values were $0.010 \%$ and $0.024 \%$ - a range 2.4 -fold. For all 50 States for 2018 the data were: $0.015 \pm 24 \%$, range $0.012-0.019,0.014$ median, and lowest and highest values were 0.0096 and 0.0261 - a range of 2.7 - fold. The flu and pneumonia data have significant variability but using them to assess health effects and to make policy decisions seems plausible.

COVID-19 deaths are reported for all 50 U.S. States ${ }^{56} 57$. The data are updated regularly and the data for June 12, 2020 as reported by COVID-19 Daily Dashboard ${ }^{58}$ were used to calculate percentage of deaths and the results were: $0.028 \pm 0.035 \%$, median $0.015 \%$; and lowest and highest values were $0.0012 \%$ and $0.157 \%$, a difference of 132 -fold. For 25 countries with the highest case counts as of June 9, 2020 values were: $6.30 \% \pm(83 \%)$, range $1.02-11.58 \%$; median $4.8 \%$. For the country with the lowest case count (Qatar) the numbers were $0.08 \%$ and highest (Belgium) $16.2 \%$, a difference of 202.5 -fold. The variability (even considering the known sources) clearly indicates the low reliability of COVID-19 data for making decisions relating to health and affecting significant social and economic policy. The data simply cannot reliably tell us how infectious or deadly COVAD-19 is. We do not know what we do not know. Science must do all it can to resolve this question.

\section{CONSCENSUS JOINT STATEMENT BY CDC AND ASPR}


The Communicable Disease and Prevention Center (CDC) and the Office of the Assistant Secretary for Preparedness and Response (ASPR) have developed five COVID-19 Pandemic Planning Scenarios that

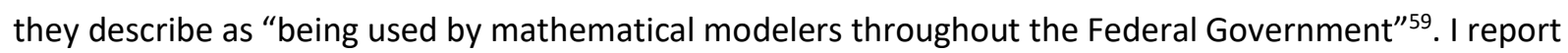
on the July update which is based on data received by the CDC through June 29, 2020 (new data are received regularly). The report states: "Many uncertainties remain..." Many examples are given. The report clearly states that the "parameters in the scenarios are not predictions of the expected effects of Covid-19... The five COVID-19 planning scenarios... represent a range of possible parameters for COVID19 in the United States".

Scenarios 1 through 4 are stated to be based on parameter values that represent the lower and upper bounds of disease severity and viral transmissibility (moderate to very high). The report states: "The parameter values used in these scenarios are likely to change as we obtain additional data about the upper and lower bounds of disease severity and the transmissibility of SARS-CoV-2". Scenario 5 represents "a current best estimate about viral transmission and disease severity in the United States with the same caveat: that the parameter values will change as more data becomes available". Scenario 5 is most relevant to this review: "Parameter values for disease severity, viral transmissibility, and presymptomatic and asymptomatic disease transmission that represent the best estimate based on the latest surveillance data and scientific knowledge Parameter values are based on data received by CDC through June 29, 2020".

For Scenario 5 (the current best estimate) the percent of infections that are asymptomatic is $40 \%$. The values for Scenarios 1-4 are, respectively, 10\%, 70\%, 10\%, 70\%. A relevant footnote states: "The percent of cases that are asymptomatic, i.e. never experience symptoms remains uncertain. Longitudinal testing of individuals over time is required to accurately indicate the absence of symptoms for the full period of infectiousness." Extensive information about method is provided and it is noted: "Studies vary in the definition of a symptomatic case... the percent of cases that are asymptomatic may vary by age and studies also vary in the age groups included." The report appropriately concludes: "Given these limitations, the range of estimates for Scenarios 1-4 is wide. The lower bound estimate approximates the lower $95 \%$ confidence interval bound estimate."

An earlier report of May 5, 2020 can be accessed in the archives at the end of the article ${ }^{59}$. As the best estimate at the time, it provides a useful comparison for asymptomatic cases. The percent of infections that are asymptomatic was stated to be $20 \%, 50 \%, 20 \%, 50 \%$, and $35 \%$, respectively, for planning scenarios 1-5. The characteristics of each scenario are detailed in the report. Scenario 5 (35\%) was described as the "Current Best Estimate". For the percentage of infections that are asymptomatic, the following is stated:

The scenarios are intended to advance public health preparedness and planning. They are not predictions or estimates of the expected impact $f$ COVID-19. The parameter values in each scenario will be updated and augmented over time, as we learn more about the epidemiology of COVID-19. Additional parameter values might be added in the future (e.g., population density, household transmission, and/or race and ethnicity). 
The description of Scenario 5 is: "Parameter values for disease severity, viral transmissibility, and presymptomatic and asymptomatic disease transmission that represent the best estimate, based on the latest surveillance data and scientific knowledge. Parameter values are based on data received by CDC prior to 4/29/2020." The contributors that differ among the scenarios include: $\mathrm{R}_{0}$ (range, 2 to 3); symptomatic case fatality ratio, stratified by age (age ranges, 0-49 years, 50-64 years, 65+ years) and an overall ratio of 0.004 for Scenario 5 . The case fatality rate is $0.4 \%$. The overall value for category 5 for the systematic case hospitalization ratio, stratified by age had an overall value for category 5 is 0.034 ; infectiousness of asymptomatic individuals relative to symptomatic individuals had a range of values from $50 \%$ or $100 \%$ with a value of $100 \%$ for Scenario 5 .

\section{SUMMARY CONCLUSION}

It is significant that the CDC best estimates for "percent of infections that are asymptomatic" published for May 20, 2020 and July 10, 2020, respectively, are 35\% and 40\%. However, four scenarios are proposed and their range of values is wide. For the earlier date values were $20 \%, 50 \%, 20 \%$, and $50 \%$ and for the latest estimate: $10 \%, 70 \%, 10 \%$, and $70 \%$.

Conflicts of Interest: The author states there are no conflicts of interest.

\section{REFERENCES}

1. Gorbalenya, A. E. et al. The species severe acute respiratory syndrome-related coronavirus : classifying 2019-nCoV and naming it SARS-CoV-2. Nat. Microbiol. 5, 536-544 (2020).

2. Zhao, H., Lu, X., Deng, Y., Tang, Y. \& Lu, J. COVID-19: asymptomatic carrier transmission is an underestimated problem. Epidemiol. Infect. 148, e116, 1-3.

https://doi.org/10.101/S0950268820001235 (2020).

3. Faranda, D. et al. Asymptotic estimates of SARS-CoV-2 infection counts and their sensitivity to stochastic perturbation. Chaos 30, 051107 doi: 10.1063/5.0008834 (2020).

4. Wang, Y., Kang, H., Liu, X. \& Tong, Z. Asymptomatic cases with SARS-CoV-2 infection. J. Med. Virol. (2020) doi:10.1002/jmv.25990. (2020).

5. Oran, D. P. \& Topol, E. J. Prevalence of Asymptomatic SARS-CoV-2 Infection. Ann. Intern. Med. (2020) doi:10.7326/M20-3012. (2020).

6. Bai, Y. et al. Presumed Asymptomatic Carrier Transmission of COVID-19. JAMA 323, 1406-1407 (2020). 
7. Nishiura, H. et al. Estimation of the asymptomatic ratio of novel coronavirus infections (COVID-19). Int. J. Infect. Dis. IJID Off. Publ. Int. Soc. Infect. Dis. 94, 154-155 (2020).

8. Hsieh, Y.-H. et al. Asymptomatic ratio for seasonal H1N1 influenza infection among schoolchildren in Taiwan. BMC Infect. Dis. 14, 80 (2014).

9. Gentile, G. \& Micozzi, A. Speculations on the clinical significance of asymptomatic viral infections. Clin. Microbiol. Infect. 22, 585-588 (2016).

10. Younes, N. et al. Challenges in Laboratory Diagnosis of the Novel Coronavirus SARS-CoV-2. Viruses 12, 582. doi: 10.3390/v12060582 (2020).

11. Chen, Y. et al. [Epidemiological characteristics of infection in COVID-19 close contacts in Ningbo city]. Zhonghua Liu Xing Bing Xue Za Zhi Zhonghua Liuxingbingxue Zazhi 41, 667-671 (2020).

12. Wu, Z. Y. [Contribution of asymptomatic and pre-symptomatic cases of COVID-19 in spreading virus and targeted control strategies]. Zhonghua Liu Xing Bing Xue Za Zhi Zhonghua Liuxingbingxue Zazhi 41, 801-805 (2020).

13. Pan, X. et al. Asymptomatic cases in a family cluster with SARS-CoV-2 infection. Lancet Infect. Dis. 20, 410-411 (2020).

14. Pan, Y. et al. Epidemiological and clinical characteristics of 26 asymptomatic SARS-CoV-2 carriers. J. Infect. Dis. (2020) doi:10.1093/infdis/jiaa205. (2020).

15. Yin G, Jin H. Comparison of Transmissibility of Coronavirus Between Symptomatic and Asymptomatic Patients: Reanalysis of the Ningbo COVID-19 Data.

https://journalclub.wustl.edu/2020/06/03/comparison-of-transmissibility-of-coronavirus-betweensymptomatic-and-asymptomatic-patients-reanalysis-of-the-ningbo-covid-19-data/ (2020).

16. Wang, Y. et al. Clinical Outcomes in 55 Patients With Severe Acute Respiratory Syndrome Coronavirus 2 Who Were Asymptomatic at Hospital Admission in Shenzhen, China. J. Infect. Dis. 221, 1770-1774 (2020). 
17. Bai, S. L. et al. [Analysis of the first cluster of cases in a family of novel coronavirus pneumonia in Gansu Province]. Zhonghua Yu Fang Yi Xue Za Zhi 54 (5):4991-493 (2020).

18. Ganyani, T. et al. Estimating the generation interval for coronavirus disease (COVID-19) based on symptom onset data, March 2020. Eurosurveillance 25 (17), 2000257 (2020).

19. Long, Q.-X. et al. Clinical and immunological assessment of asymptomatic SARS-CoV-2 infections. Nat. Med. (2020) doi:10.1038/s41591-020-0965-6. (2020).

20. Lowth, M. \& Jarvis, S. Coronavirus: what are asymptomatic and mild COVID-19? https://patient.info/news-and-features/coronavirus-what-are-asymptomatic-and-mild-covid-19. (2020).

21. Kong, W. et al. Comparison of clinical and epidemiological characteristics of asymptomatic and symptomatic SARS-CoV-2 infection: A multi-center study in Sichuan Province, China. Travel Med. Infect. Dis. 101754 (2020) doi:10.1016/j.tmaid.2020.101754. (2020).

22. Gudbjartsson, A. et al. Spread of SARS-CoV-2 in the Icelandic Population. NEJM. https://www-nejmorg.proxy.mul.missouri.edu/doi/full/10.1056/NEJMoa2006100. (2020).

23. Lavezzo, et al. Suppression of the COVID-19 outbreak in the municipality of Vò, Italy. CEBM https://www.cebm.net/study/suppression-of-the-covid-19-outbreak-in-the-municipality-of-voitaly/. (2020).

24. Hu, Z. et al. Clinical characteristics of 24 asymptomatic infections with COVID-19 screened among close contacts in Nanjing, China. Sci. China Life Sci. 63, 706-711 (2020).

25. Lai, C.-C. et al. Asymptomatic carrier state, acute respiratory disease, and pneumonia due to severe acute respiratory syndrome coronavirus 2 (SARS-CoV-2): Facts and myths. J. Microbiol. Immunol. Infect. 53, 404-412 (2020).

26. Liu, L. et al. Optimizing screening strategies for coronavirus disease 2019: A study from Middle China. J. Infect. Public Health 13, 868-872 (2020). 
27. Kim, S. E. et al. Viral kinetics of SARS-CoV-2 in asymptomatic carriers and presymptomatic patients. Int. J. Infect. Dis. 95, 441-443 (2020).

28. Chen, M. et al. A SARS-CoV-2 familial cluster infection reveals asymptomatic transmission to children. J. Infect. Public Health 13, 883-886 (2020).

29. Qiu, H. et al. Clinical and epidemiological features of 36 children with coronavirus disease 2019 (COVID-19) in Zhejiang, China: an observational cohort study. Lancet Infect. Dis. 20, 689-696 (2020).

30. Moriarty, L. F. Public Health Responses to COVID-19 Outbreaks on Cruise Ships - Worldwide, February-March 2020. MMWR Morb. Mortal. Wkly. Rep. 69, (2020).

31. Mizumoto, K. et al. Estimating the asymptomatic proportion of coronavirus disease 2019 (COVID-19) cases on board the Diamond Princess cruise ship, Yokohama, Japan, EuroSurveill 2020:25(10):2000180 (2020).

32. Sakurai, A. et al. Natural History of Asymptomatic SARS-CoV-2 Infection. N. Engl. J. Med. nejm.org (2020).

33. Ing, A. J., Cocks, C. \& Green, J. P. COVID-19: in the footsteps of Ernest Shackleton. Thorax (2020) doi:10.1136/thoraxjnl-2020-215091. (2020).

34. Payne, D. C. SARS-CoV-2 Infections and Serologic Responses from a Sample of U.S. Navy Service Members - USS Theodore Roosevelt, April 2020. MMWR Morb. Mortal. Wkly. Rep. 69, (2020).

35. Le porte-avions Charles-de-Gaulle. LExpress.fr https://www.lexpress.fr/actualite/monde/le-porteavions-charles-de-gaulle_1738857.html (2015).

36. Lytras, T. et al. High prevalence of SARS-CoV-2 infection in repatriation flights to Greece from three European countries. J. Travel Med. 27, 1-2. doi:10.1093/jlm/taaa05 (2020).

37. Nicastri, E. et al. Coronavirus disease (COVID-19) in a paucisymptomatic patient: epidemiological and clinical challenge in settings with limited community transmission, Italy, February 2020. Euro 
Surveill. Bull. Eur. Sur Mal. Transm. Eur. Commun. Dis. Bull. 25, (11):

pi=2000230.https://doi.org/10.2807/1560-7917.ES.2020.25.112000230 (2020).

38. Gostic, K., Gomez, A. C., Mummah, R. O., Kucharski, A. J. \& Lloyd-Smith, J. O. Estimated effectiveness of symptom and risk screening to prevent the spread of COVID-19. elife 9:e55570, (2020).

39. Baggett, T. P., Keyes, H., Sporn, N. \& Gaeta, J. M. Prevalence of SARS-CoV-2 Infection in Residents of a Large Homeless Shelter in Boston. JAMA 323, 2191-2192 (2020).

40. Chou, E. Dozens positive for coronavirus at LA's Skid Row homeless shelter, after all residents tested. Daily News https://www.dailynews.com/dozens-positive-for-coronavirus-at-las-skid-rowhomeless-shelter-after-all-residents-tested (2020).

41. Sutton, D., Fuchs, K., D'Alton, M. \& Goffman, D. Universal Screening for SARS-CoV-2 in Women Admitted for Delivery. N. Engl. J. Med. 382, 2163-2164 (2020).

42. Rivett, L. et al. Screening of healthcare workers for SARS-CoV-2 highlights the role of asymptomatic carriage in COVID-19 transmission. eLife 9: e58728.https://doi.org/10.7554.elife.58728 (2020).

43. Arons, M. M. et al. Presymptomatic SARS-CoV-2 Infections and Transmission in a Skilled Nursing Facility. N. Engl. J. Med. 382, 2081-2090 (2020).

44. Kimball, A. Asymptomatic and Presymptomatic SARS-CoV-2 Infections in Residents of a Long-Term Care Skilled Nursing Facility - King County, Washington, March 2020. MMWR Morb. Mortal. Wkly. Rep. 69 (13) April 3 (2020).

45. In Four U.S. State Prisons, Nearly 3,300 Inmates Test Positive for Coronavirus -- 96\% Without Symptoms. US News \& World Report https://www.usnews.com/news/top-news/articles/2020-0425/in-four-us-state-prisons-nearly-3-300-inmates-test-positive-for-coronavirus-96-withoutsymptoms. (2020). 
46. San Francisco testing blitz shows Covid-19 hit mostly low-wage workers. STAT https://www.statnews.com/2020/05/28/sobering-finding-covid19-struck-mostly-low-wageessential-workers-san-francisco/ (2020).

47. Souza, T. H. de, Nadal, J. A., Nogueira, R. J. N., Pereira, R. M. \& Brandão, M. B. Clinical manifestations of children with COVID-19: A systematic review. Pediatr. Pulmonol.1-8. DOI: 10.1002/ppul.24885. (2020).

48. Shelmerdine, S. C. et al. Coronavirus disease 2019 (COVID-19) in children: a systematic review of imaging findings. Pediatr. Radiol. (2020) doi:10.1007/s00247-020-04726-w. (2020).

49. Lan, L. et al. Early CT Findings of Coronavirus Disease 2019 (COVID-19) in Asymptomatic Children: A Single-Center Experience. Korean J. Radiol. 21, 919 doi.org/10.3348/kjr.2020.0231 (2020).

50. NIH begins study to quantify undetected cases of coronavirus infection. National Institutes of Health (NIH) https://www.nih.gov/news-events/news-releases/nih-begins-study-quantify-undetectedcases-coronavirus-infection (2020).

51. Viboud, C. et al. Global Mortality Impact of the 1957-1959 Influenza Pandemic. J. Infect. Dis. 213, 738-745 (2016).

52. Hayward, A. C. et al. Comparative community burden and severity of seasonal and pandemic influenza: results of the Flu Watch cohort study. Lancet Respir. Med. 2, 445-454 (2014).

53. Horby, P. W. Community studies of influenza: new knowledge, new questions. Lancet Respir. Med. 2, 430-431 (2014).

54. Belongia, E. A. \& Osterholm, M. T. COVID-19 and flu, a perfect storm. Science 368, 1163-1163 (2020).

55. Stats of the States - Influenza/Pneumonia Mortality. https://www.cdc.gov/nchs/pressroom/sosmap/flu_pneumonia_mortality/flu_pneumonia.htm (2020). 
56. U.S. COVID-19 deaths by state. Statista https://www.statista.com/statistics/1103688/coronaviruscovid19-deaths-us-by-state/. (2020).

57. COVID-19 Death Data and Resources - National Vital Statistics System. https://www.cdc.gov/nchs/nvss/covid-19.htm (2020).

58. COVID-19: Daily dashboard. https://dgalerts.docguide.com/covid-19-daily-dashboard-74.

59. CDC. Coronavirus Disease 2019 (COVID-19). Centers for Disease Control and Prevention https://www.cdc.gov/coronavirus/2019-ncov/hcp/planning-scenarios.html (2020). 